\title{
Research on the ESXI Host Renovation Project based on VMware vCenter
}

\author{
Kun $\mathrm{Wu}^{\mathrm{a}}$, Bao Wang ${ }^{\mathrm{b}}$ \\ CIO Organization of BOE Technology Group Co., Ltd., Beijing, China. \\ awuk@boe.com.cn, bwangbao@boe.com.cn
}

\begin{abstract}
Many enterprises currently undertake part of their production system by deploying virtual resources on a single server (local mode). However, with the increase of business pressure and the aging of equipment, the disadvantages of system performance are more and more obvious. The single-machine mode is difficult to load and cannot meet the enterprise's requirements for high availability. Based on this background, this paper proposes to use VMware vCenter technology to transform the single-machine virtual environment, so as to provide more flexible and secure virtual environment support.
\end{abstract}

Keywords: ESXI; VMware Vcenter; Virtual.

\section{Introduction}

With the constant improvement of enterprise informatization, the function of information system is more and more comprehensive. Many operating departments develop their own systems, such as department sharing, access management system, administrative system and so on. These systems require more and more resources, so how to provide stable and highly available server resources has become an urgent problem for IT departments.

In the past, we mostly adopted the single-machine single-use mode, that is, one server resource is used for each business system separately, so the boundary is clear and accurate positioning can be achieved for the failure of the business system. However, this mode has a single point of risk, with high risk of hardware failure and data loss, which makes it difficult to ensure the high availability of business. On the other hand, the stand-alone mode cannot achieve the maximum utilization of resources, and the equipment with higher-configured may be used for the smaller requirements, and the optimized allocation of resources cannot be realized better.

Therefore, we started to try to build ESXI host to create virtual machines to meet business demands. However, due to the gradual growth of business and the lack of pre-planning, problems such as unreasonable use of ESXI host resources and reduced availability were easily caused. VMware vCenter technology can solve such problems and provide us with new ideas for re-planning equipment resource allocation. Organization of the Text.

\section{Related Technical Introduction}

ESXI is a virtualized management program, which can be directly installed on the physical server to run the virtual machine and realize the rapid installation, configuration and deployment of multiple systems of the virtual machine. Under the same ESXI architecture, multiple virtual machines run simultaneously and share the resources of the physical server. Using ESXI architecture, it can realize dynamic adjustment of CPU, memory, hard disk and other resources, improve utilization rate of hardware and simplify management. Therefore, ESXI architecture is widely used in enterprise applications.

VMware HA(High Availability), is a functional component of VMware vSphere to achieve business continuity. It uses multiple ESXI hosts to be configured into cluster mode, and vCenter server will manage cluster resources and configuration to improve the high availability of the entire cluster.

VMware vCenter server is the foundation of virtualization management. With vCenter, it can conveniently implement the functions, such as vMotion (online migration), DRS (distributed resource 
scheduler), HA (high availability), FT (fault tolerance) which improves the control of administrators over the entire virtual environment.

\section{Stand-alone Mode Introduction}

The architecture of single-machine mode is shown in figure 1. All the three servers have installed ESXI system, and they are independent and do not share resources with each other. Each host virtual out multiple virtual host environments, install corresponding operating systems, and carry different businesses.

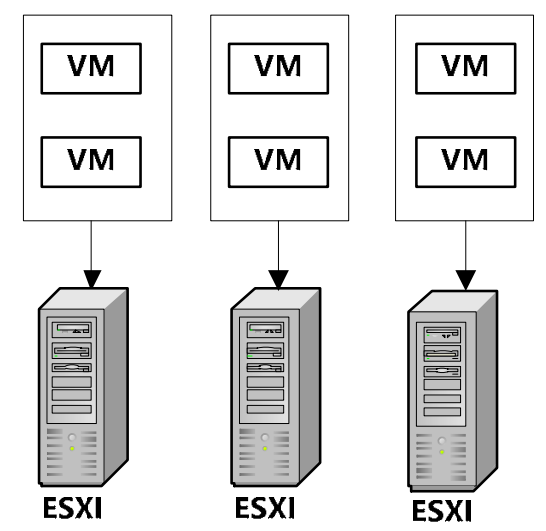

Figure 1. The architecture of single-machine mode

The advantage of this architecture is that it is more convenient to use, the server is allocated according to demand, and the single server is more flexible, but the disadvantages are also very obvious:

(1) The single point of fault risk is high. If a host fails, the VM running on it cannot be used normally;

(2) The server uses local disks, which have a higher failure rate and lower data reading efficiency;

(3) It is difficult to expand. If the current host cannot meet the business demand, all the service systems on the host should be shut down for hardware expansion;

(4) The omnibus load is unbalanced, the VM load of each ESXI host is inconsistent, and the resource allocation is unbalanced, resulting in the continuous high load of some ESXI hosts;

\section{HA Renovation Project}

Our renovation project is to introduce the VMware HA mechanism to achieve high availability and load balancing of all hosts in the cluster. A storage device is needed here. Only ESXI is installed on the host hard disk, while the VM data is stored in storage. The architecture is shown in figure 2.

As shown in figure 2, three ESXI servers create a cluster, when one of the hosts fails (ESXI2 in figure 2), the virtual machines running on it can be automatically migrated to other hosts in the cluster (VM2.1 migrated to ESXI1, VM 2.2 migrated to ESXI3 in figure 2). Manual migration can also be carried out according to the load condition. And online migration does not affect the running of business systems on the VM.

After the renovation, the cluster performance has been significantly improved, mainly in the following aspects:

(1) High security. Using storage devices to provide space, performance is improved significantly, redundancy is higher, and data is more secure;

(2) Management is easier. it is more convenient to manage the resources in the cluster through the Vcenter without login to the three Vsphere management interfaces; 


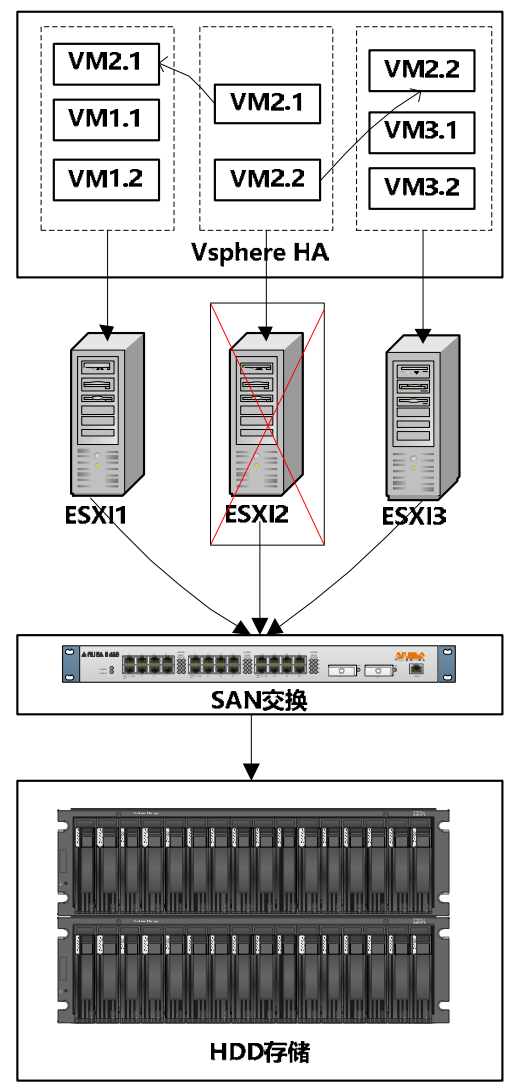

Figure 2. The architecture of HA mode

(3) Resources are used more flexible. Shared memory is flexible to use. And migrate VM online to balance CPU and memory resources;

(4) Expansion facilities. Storage can be directly extended hard disk. Server hardware can expansion after migration VM online, can also directly added new servers to the cluster. The whole expansion process does not affect the operation of the VM.

The renovation of vCenter greatly shortens the failure response time in cluster. Response time of VM reboot on other hosts in the cluster: VM system startup time + application startup time + heartbeat detection time of about 15 seconds. Usually this time can be kept within 3 minutes.

\section{Conclusion}

Virtualization technology is becoming more mature and widely used. VCenter manages VMware vSphere environment to realize optimal allocation of cluster resources, while VSAN technology is a distributed storage architecture based on vSphere. In our subsequent projects, we will also review VSAN technology.

\section{References}

[1]. Information on: https://www.vmware.com/products/vcenter-server.html.

[2]. Anita Choudhary, Mahesh Chandra Mahesh Chandra Govil, Girdhari Singh, Lalit K. Awasthi, Emmanuel S. Pilli, Divya Kapil. A critical survey of live virtual machine migration techniques. Journal of Cloud Computing, 2017, Vol.6 (1), pp.1-41.

[3]. Y Li,H Yang.Virtualization construction of university data center based on vSphere, Experimental Technology \& Management, 2018.

[4]. Information on: http://blog.51cto.com/wzlinux/2094598. 